\title{
Effect of combination therapy of arthrocentesis and occlusal splint on nonreducing temporomandibular joint disk displacement
}

\author{
Peter Tvrdy ${ }^{\mathrm{a}}$, Petr Heinz ${ }^{\mathrm{a}}$, Jana Zapletalova ${ }^{\mathrm{b}}$, Richard Pink ${ }^{\mathrm{a}}$, Petr Michla
}

\begin{abstract}
Objective. Arthrocentesis of the temporomandibular joint is a minimally invasive method of treatment located at the boundary between conservative and surgical therapy. It is usually performed on an out-patient basis under local anaesthesia. These days, arthrocentesis of the temporomandibular joint is used not only in cases of acute closed lock but also in the treatment of various temporomandibular disorders. The most frequent indication is acute anterior displacement of the articular disc without reduction. Treatment using occlusal splint is one of the most frequently used methods of conservative treatment. It is used mainly in the case of discopathies and myofascial pain.

Aim. The aim of the study was to confirm that simultaneous use of the occlusal splint and arthrocentesis makes the treatment more effective in the case of detected disc dislocation without reduction.

Materials, Methods and Results. From 2008 to 2013, 144 patients underwent arthrocentesis simultaneously using occlusal splint in the treatment of chronic closed lock. The study group consisted of a 130 (90.3\%) women and 14 (9.7\%) men. After 3 months of therapy, a good treatment outcome was found in 98 (68.1\%) patients, $12(85.7 \%)$ men and 86 (66.2\%) women. The treatment did not have any effect in 46 (31.9\%) patients, 2 (14.3\%) men and 44 (33.8\%) women. Conclusion. The simultaneous use of the occlusal splint and arthrocentesis makes the treatment more effective in the case of detected joint disc dislocation without reduction.
\end{abstract}

Key words: temporomandibular joint disorder, arthrocentesis, splint therapy

Received: April 9, 2014; Accepted: July 18, 2014; Available online: August 18, 2014

http://dx.doi.org/10.5507/bp.2014.044

${ }^{a}$ Department of Oral and Maxillofacial Surgery, University Hospital Olomouc, Czech Republic ${ }^{b}$ Department of Biophysics, Faculty of Medicine and Dentistry, Palacky University Olomouc Corresponding author: Richard Pink, e-mail: richard.pink@seznam.cz

\section{INTRODUCTION}

The main goal of temporomandibular joint disorder (TMD) treatment is to alleviate pain and reduce functional disorder of the joint. TMD treatments can be divided into two categories: conservative and surgical methods. Conservative therapy consists in the administration of analgesic and anti-inflammatory medication and the use of physical therapy and treatment by means of bite splints (splint therapy). Surgical treatment comprises mini-invasive methods, which include arthrocentesis and arthroscopy or the open surgical approach. Conservative, reversible methods are widely accepted as the first choice for TMJ treatment ${ }^{1}$. Therapy by means of the occlusal splint is one of the most frequently used conservative treatments. It is used mainly in the case of discopathies and myofascial pain. As for the mechanisms by which the occlusal splint acts, it is assumed that the occlusal splint alters the action of sensitive effects originating in periodontal tissues and afferent fibres of the muscles of mastication $^{2}$. At the same time, it reduces intra-articular pressure ${ }^{3}$. Frequently, therapy by means of the occlusal splint is successful; however, it requires long-term application and considerable patience on the part of the patient.

As for surgical methods, the first-choice method is a mini-invasive approach - arthrocentesis. It is a gentle method for lavage of the joint space, which is generally indicated for patients in acute closed lock or in the case of poor response to conservative therapy ${ }^{4}$. Arthrocentesis of the temporomandibular joint was first described by D. W. Nitzan in 1991 as the simplest form of surgical therapy with the objective of washing out inflammatory mediators, releasing the articular disc, and disrupting adhesions between the surface of the disc and the articular fossa by hydraulic pressure of the lavage solution ${ }^{5}$.

The aim of our study was to verify the hypothesis that the simultaneous use of the occlusal splint and arthrocentesis makes the treatment more effective in the case of detected disc dislocation without reduction.

\section{MATERIALS AND METHODS}

We enrolled 144 patients in the study; they were treated in 2008-2013 at the Department of Oral and Maxillofacial Surgery, University Hospital Olomouc and Faculty of Medicine and Dentistry, Palacky University Olomouc with diagnosed articular disc dislocation without reduction. The diagnosis was determined from a clinical examination and an examination by means of magnetic resonance imaging (MRI). Additionally, patient selection was contingent on the same case history data: restricted range of mouth opening, pain in the preauricular area, problems with only one TMJ, and at least a 
three-month duration of the problems. The following were excluded: patients with systemic disease or degenerative joint impairment, patients after trauma, and patients with the anomalies of the face and jaw.

The patients underwent combined treatment consisting of arthrocentesis and TMJ lavage with subsequent application of a repositioning bite splint.

Before initiating treatment, we measured the range of mouth opening between the incisal edges of the upper and lower incisors. This figure was identified as maximal mouth opening (MMO). Other input data consisted in assessing pain using the visual analogue scale (VAS). On a 0-10 scale, the patients marked the level of their pain, with 0 representing no pain, and 10 representing maximum pain.

The same measurements were carried out 3 months after treatment. The success of the treatment was evaluated according to the AAOMS criteria: no pain or only mild pain and, at the same time, the range of mouth opening exceeding $35 \mathrm{~mm}$.

Statistical data analysis was conducted using the SPSS software, version 15 (SPSS Inc., Chicago, USA), in collaboration with the Department of Biophysics, Faculty of Medicine Olomouc. The Wilcoxon matched pairs test was used to compare the measured parameters before treatment and after 3 months of treatment. Spearman's correlation analysis was used to evaluate the effect of the change in the measured parameters on age and the MannWhitney U test was used to evaluate the effect of change in parameters with respect to gender. A group of patients

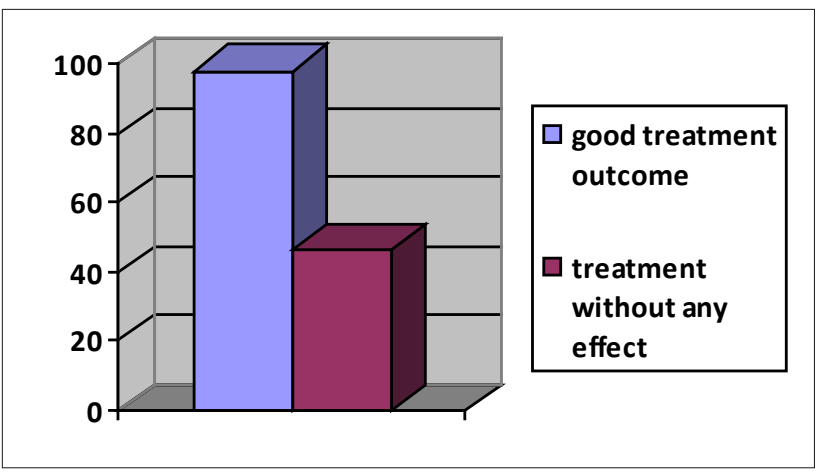

Fig. 1. Good treatment outcome (in total).

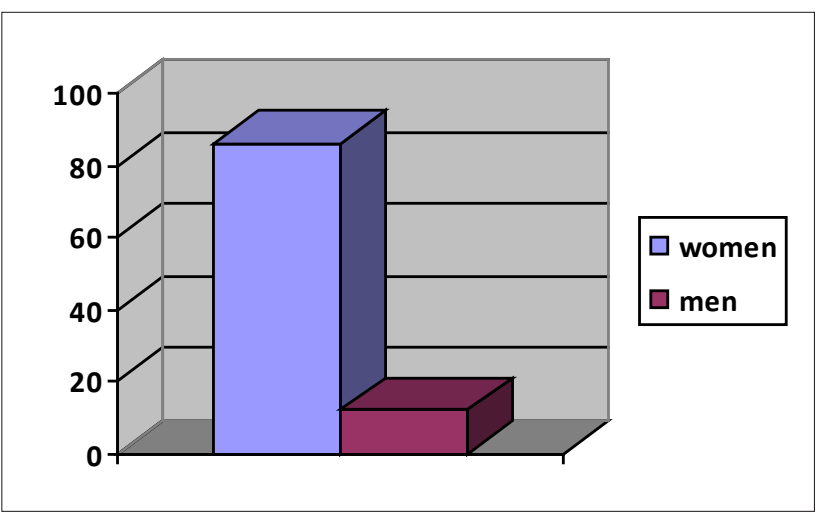

Fig. 2. Good treatment outcome (men/women). exhibiting a good treatment outcome was compared with a group exhibiting no treatment effect, with regard to age, the values of the studied parameters measured before treatment and after treatment, and the change in parameter values determined by the Mann-Whitney U test. Fisher's exact test was used to compare the distribution by gender. The normality of the data was verified using the Shapiro-Wilk test. The tests were performed using a significance level of 0.05 .

In all patients, treatment was initiated using arthrocentesis and lavage of the upper joint space by means of a generally known method. After a peripheral block of the auriculotemporal nerve using an injection of a local anaesthetic, an injection needle was inserted into the location of a connecting line between the tragus and the outer corner of the eye (the Holmlund-Hellsing line), approximately $1 \mathrm{~cm}$ anterior to the tragus in the area of the articular fossa ${ }^{6,7}$. To distend the joint space, 1-2 mL of physiological saline or Ringer's solution was used. Subsequently, a second injection needle was inserted into the site of the articular eminence. Using the first needle, a lavage solution was then instilled under continuous flow and allowed to drain continuously through the second needle ${ }^{8,9}$.

In the next step, the patient wore a repositioning occlusal splint in the mouth. In our department, we use a hard acrylic splint for the lower jaw, with a 4-mm width, which is produced so that the lower jaw is moved into a slight protrusion. Patients wear the splint daily for 3-5 months, for an 8-hour period but not at night.

\section{RESULTS}

The study group consisted of a total of 144 patients, $130(90.3 \%)$ women and $14(9.7 \%)$ men. The median age was 26.5 (16-50 years). The median age of the women was 26.0 (16-50 years) and the median age of the men was 28.5 ( $18-46$ years).

After 3 months of treatment, a good treatment outcome was noted in $98(68.1 \%)$ patients, $12(85.7 \%)$ men and $86(66.2 \%)$ women. The treatment had no effect in 46 (31.9\%) patients, 2 (14.3\%) men and 44 (33.8\%) women. (Fig. 1-3).

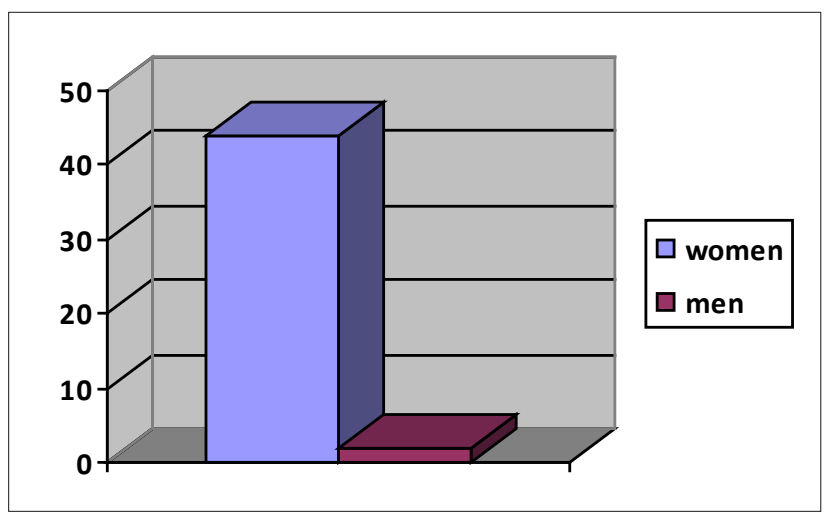

Fig. 3. Treatment without any effect (men/women). 
Table 1. Evaluation of the significance of the change in the parameters after treatment.

\begin{tabular}{lcccc}
\hline & $\begin{array}{c}\text { Before } \\
\text { treatment }\end{array}$ & $\begin{array}{c}\text { 3 months } \\
\text { after treatment }\end{array}$ & $\begin{array}{c}\text { Change after 3 months } \\
\text { (3 months - before treatment) }\end{array}$ & $P$ \\
\hline MMO $(\mathrm{mm})$ & $27.0(16-37)$ & $40.0(20-57)$ & $13.0(-3$ to +28$)$ & $<0.0001$ \\
VAS & $7(4-10)$ & $2(0-10)$ & $-4(-9$ to +3$)$ & $<0.0001$ \\
\hline
\end{tabular}

Median (minimum-maximum), $P$ - significance of the Wilcoxon matched pairs test

Table 2. A comparison of patients with a good treatment outcome vs. without any treatment effect.

\begin{tabular}{lccc}
\hline & $\begin{array}{c}\text { Good treatment outcome } \\
(\mathrm{n}=98)\end{array}$ & $\begin{array}{c}\text { Treatment without any effect } \\
\text { (n= 46) }\end{array}$ & $P$ \\
\hline Gender & $86 / 12$ & $44 / 2$ & $0.226^{\mathrm{a}}$ \\
women/men & $(88 \% / 12 \%)$ & $(96 \% / 4 \%)$ & $0.842^{\mathrm{b}}$ \\
Age (years) & $27.0(16-46)$ & $25.0(17-50)$ & $<0.0001^{\mathrm{b}}$ \\
Change in MMO (mm) (after - before treatment) & $16.0(4-28)$ & $2.0(7$ to -3$)$ & $0(-4$ to +3$)$ \\
Change in VAS (after - before treatment) & $-5(-2$ to -9$)$ & $0.0001^{\mathrm{b}}$ \\
\hline
\end{tabular}

Median (minimum-maximum), ${ }^{\text {a } F i s h e r ' s ~ e x a c t ~ t e s t, ~}{ }^{\mathrm{b}}$ Mann-Whitney U test

After 3 months of treatment, there was a significant increase $(P<0.0001)$ in the MOU parameter and a significant decrease $(P<0.0001)$ in the VAS parameter (Table 1$)$.

Patients with a good treatment outcome and patients without any treatment effect did not differ significantly in age $(P=0.842)$ or gender $(P=0.226)$ (Table 2$)$.

Spearman's correlation analysis did not show any significant effect of the change in MMO and the change in VAS on the patients' age $(r=-0.002$ and $r=-0.045$, respectively).

No significant effect of the change in MMO or the change in VAS on the patient's gender was found either ( $P=0.613$ and $P=0.438$, respectively).

The change in MMO and the change in VAS correlated significantly $(\mathrm{r}=-0.693 ; P<0.0001)$ (Fig. 4).

\section{DISCUSSION}

The occlusal splint is one of the most widely used methods of TMD treatment. Carraro and Caffesse argued that the application of an occlusal splint reduces TMJ pain by about $70 \%$ (ref. $^{10}$ ). However, Major and Nebbe reported that the use of the occlusal splint leads to an effective reduction of headache and muscle pain; however, it has a limited effect on reducing TMJ pain ${ }^{11}$. According to Lundh et al., occlusal splints do not have any effect in the treatment of disc dislocation without reduction ${ }^{12}$. For this reason, the treatment of TMD by means of conservative therapy, such as the occlusal splint fails, in some cases, to produce a satisfactory outcome. In 1986, Sanders described, in a case study, a successful treatment of synovitis using TMJ arthrocentesis ${ }^{13}$. Arthrocentesis began to develop and entered into common practice especially due to the efforts of Murakami et al. in 1987 and Nitzan et al. in 1990 - as a treatment option with a very satisfactory $\operatorname{prognosis}^{14,15}$.

Arthrocentesis is a minimally invasive procedure that can be performed under local anaesthesia with or without

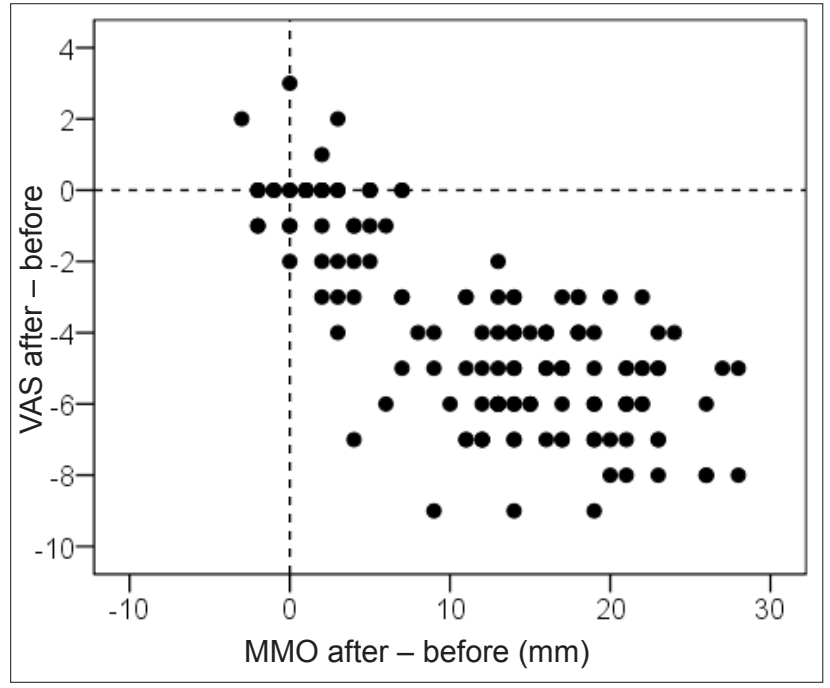

Fig. 4. Point scatter plot, change in MMO vs. change in VAS.

sedation. Its main aim is to clear the joint space of blood, loose particles, inflammatory mediators and pain mediators. In addition, it is possible to lyse any possible adhesions and to release the joint disc by hydraulic pressure. In 1999, Kropmans et al. examined the results of 62 previous studies and reported that a combination therapy using arthrocentesis and the occlusal splint was very effective ${ }^{16}$. In 2011, authors Machoň and Hirjak also reached the same conclusion in a study on the use of arthrocentesis and occlusal splint therapy in patients with osteoarthritis of the TMJ (ref. ${ }^{17}$ ). We based our study on these conclusions as well. We worked on the assumption that in the case of disc dislocation without reposition, it is important to begin with the release of the joint disc by arthrocentesis and proper lavage of the joint space, and in the case of success, to maintain this state by applying a repositioning occlusal splint, when there is alteration in the action of sensitive effects originating in periodontal tissues of the 
jaws and afferent fibres of the muscles of mastication. At the same time, intraarticular pressure is reduced.

\section{CONCLUSION}

In our sample of 144 patients, we achieved $68.1 \%$ success rate, without any significant effect of age or gender. All persons had chronic disc dislocation. In our experience, arthrocentesis is very successful and usually sufficient in acute closed lock lasting up to 14 days. Our study confirmed that for longer-lasting problems, a combination therapy is an effective method with a minimal degree of invasiveness. Patients who had no benefit from this treatment underwent arthroscopy of the TMJ.

The simultaneous use of occlusal splint and arthrocentesis makes the treatment more effective in the case of detected joint disc dislocation without reduction.

Author contributions: PT: manuscript writing; PH, RP: data collection; JZ: statistical analysis; PM: literature search.

Conflict of interest statement: None declared.

\section{REFERENCES}

1. Hye-Sung L, Hyun-Su B, Dong-Suk S, Hee-Chul K. Effect of simultaneous therapy of arthrocentesis and occlusal splints on temporomandibular disorders: anterior disc displacement without reduction. J Korean Assoc Oral Maxillofac Surg 2013;39(1):14-20.

2. Yustin $D$, Neff $P$, Rieger MR, Hurst T. Characterization of 86 bruxing patients with long-term study of their management with occlusal devices and other forms of therapy. J Orofac Pain 1993;7:54-60.
3. Nitzan DW. Intraarticular pressure in the functioning human temporomandibular joint and its alteration by uniform elevation of the occlusal plane. J Oral Maxillofac Surg 1994;52:671-9.

4. Barkin S, Weinberg S. Internal derangements of the temporomandibular joint: the role of arthroscopic surgery and arthrocentesis. J Can Dent Assoc 2000;66:199-203.

5. Nitzan DW, Dolwick MF, Martinez GA. Temporomandibular joint arthrocentesis: a simplified treatment for severe, limited mouth opening. J Oral Maxillofac Surg 1991;49:1163-7.

6. Holmlund A, Hellsing G. Arthroscopy of the temporomandibular joint. An autopsy study. Int J Oral Surg 1985;14:169-75.

7. McCain JP. Arthroscopy of the human temporomandibular joint. J. Oral Maxillofacial Surg 1988;46:648-55.

8. Monje-Gil F, Nitzan D, Gonzales-Garcia R. Temporomandibular joint arthrocentesis. Review of the literature. Med Oral Patol Oral Cir Bucal 2012;17(4),575-81.

9. Tozoglu S, Al- Belasy FA, Dolwick MF. A review of techniques of lysis and Lavage of the TMJ. Brit J Oral Maxillofac Surg 2011;49(4),302-9.

10. Carraro JJ, Caffesse RG. Effect of occlusal splints on TMJ symptomatology. J Prosthet Dent 1978;40:563-6.

11. Major PW, Nebbe B. Use and effectiveness of splint appliance therapy: review of literature. Cranio 1997;15:159-66.

12. Lundh $\mathrm{H}$, Westesson PL, Eriksson L, Brooks SL. Temporomandibular joint disk displacement without reduction. Treatment with flat occlusal splint versus no treatment. Oral Surg Oral Med Oral Pathol 1992;73:655-8

13. Sanders B. Arthroscopic surgery of the temporomandibular joint: treatment of internal derangement with persistent closed lock. Oral Surg Oral Med Oral Pathol 1986;62:361-72.

14. Murakami KI, lizuka T, Matsuki M, Ono T. Recapturing the persistent anteriorly displaced disk by mandibular manipulation after pumping and hydraulic pressure to the upper joint cavity of the temporomandibular joint. Cranio 1987;5:17-24.

15. Nitzan DW, Dolwick MF, Heft MW. Arthroscopic lavage and lysis of the temporomandibular joint: a change in perspective. J Oral Maxillofac Surg 1990;48:798-801.

16. Kropmans TJ, Dijkstra PU, Stegenga B, de Bont LG. Therapeutic outcome assessment in permanent temporomandibular joint disc displacement. J Oral Rehabil 1999;26:357-63.

17. Machon V, Hirjak D, Lukas J. Therapy of the osteoarthritis of the temporomandibular joint. J Craniomaxillofacial Surg 2011;39(2):127-30. 